\title{
Low temperature behavior of magnetic domains observed using a magnetic force microscope
}

\author{
S. H. Chung ${ }^{\text {a) }}$ \\ Department of Physics, University of Maryland, College Park, Maryland 20742 \\ and Laboratory for Physical Sciences, College Park, Maryland 20740
}

S. R. Shinde, S. B. Ogale, T. Venkatesan, and R. L. Greene

Department of Physics, University of Maryland, College Park, Maryland 20742

M. Dreyer and R. D. Gomez

Department of Electrical and Computer Engineering, University of Maryland, College Park, Maryland 20742 and Laboratory for Physical Sciences, College Park, Maryland 20740

\begin{abstract}
A commercial atomic force microscope/magnetic force microscope (MFM) was modified to cool magnetic samples down to around $100 \mathrm{~K}$ under a high vacuum while maintaining its routine imaging functionality. MFM images of a $120 \mathrm{~nm}$ thick $\mathrm{La}_{0.7} \mathrm{Ca}_{0.3} \mathrm{MnO}_{3}$ film on a $\mathrm{LaAlO}_{3}$ substrate at low temperature show the paramagnetic-to-ferromagnetic phase transition. Evolution of magnetic domains and magnetic ripples with decreasing temperature are also observed near the edge of a 20 $\mathrm{nm}$ thick patterned Co film on a Si substrate. (C) 2001 American Institute of Physics.
\end{abstract}

[DOI: $10.1063 / 1.1360262]$

\section{INTRODUCTION}

Low temperature magnetic force microscopy (MFM) has been a recent interest for the study of low temperature magnetism such as the ferromagnetic phase transition of colossal magnetoresistive (CMR) materials ${ }^{1}$ and vortex structures of high $T_{c}$ superconductors. ${ }^{2-4}$ Operating at low temperatures is very useful because it increases sample stability and decreases piezo creep. Most of the samples become more rigid and less affected by the lateral motion of the MFM tip at low temperatures. The signal-to-noise ratio also improves as thermal fluctuation and phonon scattering is reduced. We can thus investigate the physical properties which may be masked by thermal excitation such as magnetic quantum tunneling or the magnetic anisotropy of small particles that are superparamagnetic at room temperature. There is also a great need to develop an instrument that is highly adaptable and whose operation can be performed routinely and has less restriction on the sample preparation.

There are some intrinsic problems in the instrumentation of low temperature atomic force microscopy (AFM). It is very important to keep the sample free from contaminants since materials with high freezing points would be frozen to screen the real features of the sample surface. Therefore a high vacuum or a water-free environment is critical for the low temperature MFM experiment. Additionally, isolating the vibration from a mechanical noise source such as boiling liquid nitrogen or liquid helium and preventing the thermal drift of piezo are also important.

In this work we report the conversion of a commercial AFM/MFM for use in low temperature experiments. We demonstrate its capability by imaging magnetic structures of a perovskite manganite and a patterned thin $\mathrm{Co}$ film on a $\mathrm{Si}$ substrate at low temperatures.

${ }^{a)}$ Electronic mail: chungsh@glue.umd.edu

\section{INSTRUMENTATION AND EXPERIMENT}

The commercial MFM used in this work is a Nanoscope III from Digital Instruments, ${ }^{5}$ in standard (Interleave ${ }^{\mathrm{TM}}$ ) mode. The MFM measures the topographic and magnetic information in two successive scans. Both commercial and homemade MFM tips were used in our experiments.

Figure 1 shows the schematic diagram of our low temperature AFM/MFM system. The commercial MFM assembly fits in a vacuum chamber $30.5 \mathrm{~cm}$ high and $20.3 \mathrm{~cm}$ in diameter. The system consists of a pumping-purging part, a vacuum measurement part, cold finger feedthrough, and electric feedthrough. The tube chamber can be pumped down to $6 \times 10^{-7}$ Torr using a turbomolecular pump and a copper cold trap. The vacuum chamber is sealed with Viton O-rings.

The temperature of the samples can be set from 100 up to $350 \mathrm{~K}$ by a temperature controller. The sample holder is attached on top of the tube piezo of the MFM by a small magnet embedded inside the piezo. Magnetic samples are attached on top of the sample holder, which is connected to fine copper braids as a heat flow conduit. The other end of

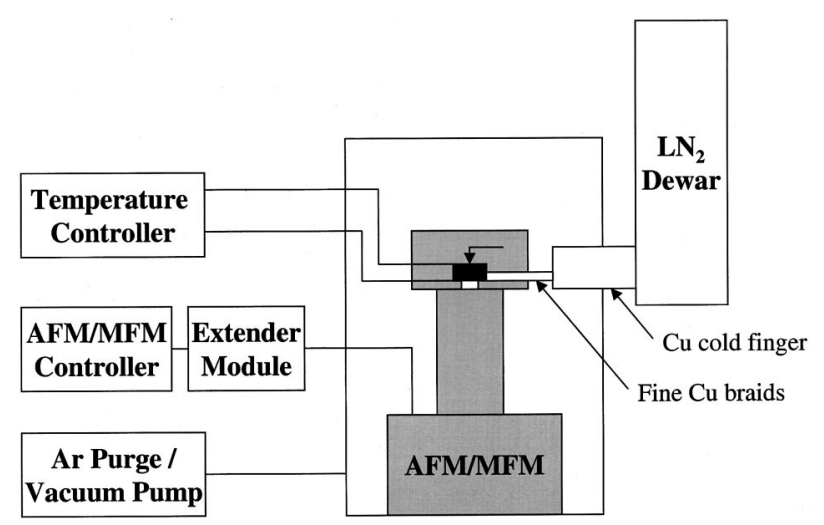

FIG. 1. Schematic diagram of the low temperature AFM/MFM system. 
the copper braid is clamped to the copper cold finger. Due to their flexibility, the fine braids reduce the transmission of mechanical vibration from boiling liquid nitrogen and the vacuum pump. The nominal noise level due to mechanical vibration is $1 \mathrm{~nm}$ and we can reduce it to less than $0.5 \mathrm{~nm}$ by increasing the length of the copper braids or by reducing the number of copper braids. The noise vibration requirement is a trade-off with the lowest attainable temperature. With 400 strands of copper ( $5 \mathrm{~cm}$ long and $0.08 \mathrm{~mm}$ in diameter), the sample can be cooled down to $100 \mathrm{~K}$ in $15 \mathrm{~min}$.

We could obtain good scan images under high and low vacuum conditions and even at a slight overpressure. Since the $Q$ factor of the MFM cantilever increases greatly with the vacuum level, the frequency resolution of an image can be enhanced under high vacuum conditions. ${ }^{6}$ A vacuum also prevents sample contamination, so we could obtain images with better quality. However, a drawback is the need to scan very slowly since it takes more time for the cantilever to reach the steady state.

\section{RESULTS AND DISCUSSION}

We first apply the technique to study the domain formation accompanying the paramagnetic-to-ferromagnetic transition of a perovskite manganites exhibiting colossal magnetoresistance (CMR). The sample is a $120 \mathrm{~nm}$ thick $\mathrm{La}_{0.7} \mathrm{Ca}_{0.3} \mathrm{MnO}_{3}$ (LCMO) film on a $\mathrm{LaAlO}_{3}(\mathrm{LAO})$ substrate. Using pulsed laser deposition, LCMO films were grown on (100) oriented single crystalline LAO. The substrate-film lattice mismatch causes in-plane compressive stress and changes in the microstructure of the film. ${ }^{7}$ The growth temperature and oxygen pressure during the deposition were kept at $820^{\circ} \mathrm{C}$ and $400 \mathrm{mTorr}$, respectively. After the deposition, the films were cooled in oxygen atmosphere.

$\mathrm{La}_{1-x} \mathrm{Ca}_{x} \mathrm{MnO}_{3}$ with $x=0.2-0.5$ exhibits a phase transition from a paramagnetic insulator to a ferromagnetic metal upon cooling, which is accompanied by a sharp drop in resistivity. ${ }^{8}$ In order to understand the physical characteristics of the sample, we performed resistivity and magnetization measurements with decreasing temperature. The magnetization was measured by a superconducting quantum interference device (SQUID) magnetometer and the resistivity was measured by the standard four-point method with indium-silver contacts. Figure 2 shows the dependence of sample resistivity $\rho$ and magnetization $M$ on temperature $T$. Without an external magnetic field, the resistivity peaks to $0.04 \Omega \mathrm{cm}$ at $255 \mathrm{~K}$ and drops greatly with decreasing temperature, approaching a nearly constant value below $200 \mathrm{~K}$. The Curie temperature $T_{c}$, determined by the maximum slope of the sudden drop, is $235 \mathrm{~K}$. On the other hand, the magnetization begins to increase at $255 \mathrm{~K}$ and saturates near $200 \mathrm{~K}$ when the magnetic field $(H=2.5 \mathrm{Oe})$ is applied parallel to the film. We note that this saturation behavior was not observed with a magnetic field perpendicular to the sample. This suggests that the magnetic easy-axis is in-plane of the sample surface.

Figures 3(a) and 3(c) are $8 \times 8 \mu \mathrm{m}^{2}$ topography of the surface of a LCMO film at room temperature and at $150 \mathrm{~K}$, respectively. The grain sizes are between 100 and $200 \mathrm{~nm}$

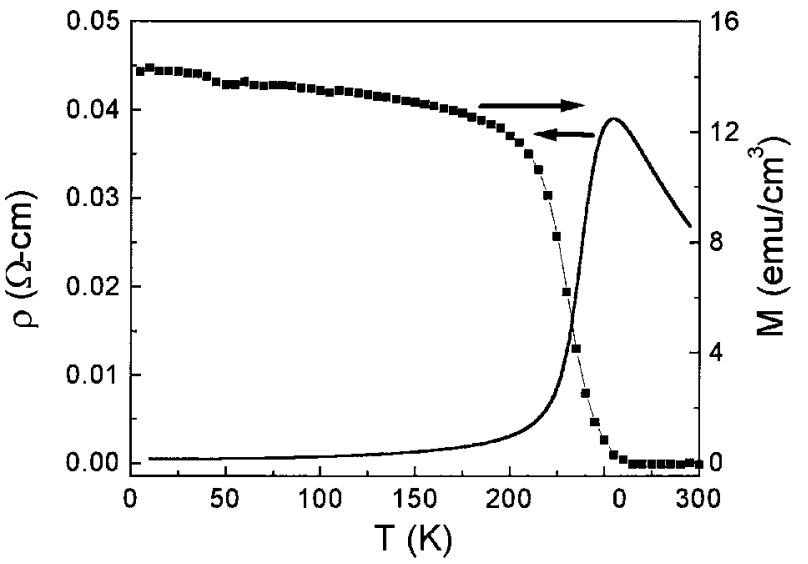

FIG. 2. Temperature dependence of resistivity $\rho$ and magnetization $M$ for a $120 \mathrm{~nm}$ thick LCMO film on a LAO substrate. The resistivity was measured without magnetic field and the magnetization was measured with $H$ $=2.5$ Oe parallel to the film.

and the surface roughness is around $8 \mathrm{~nm}$. The image quality at low and room temperature is identical and devoid of thermal drifts and condensates that could form at low temperatures. Moreover, the lateral and vertical calibrations are unaffected by extreme changes in the operating temperature.

A comparison between Figs. 3(b) and 3(d) shows quite a remarkable ferromagnetic transition of CMR material at low
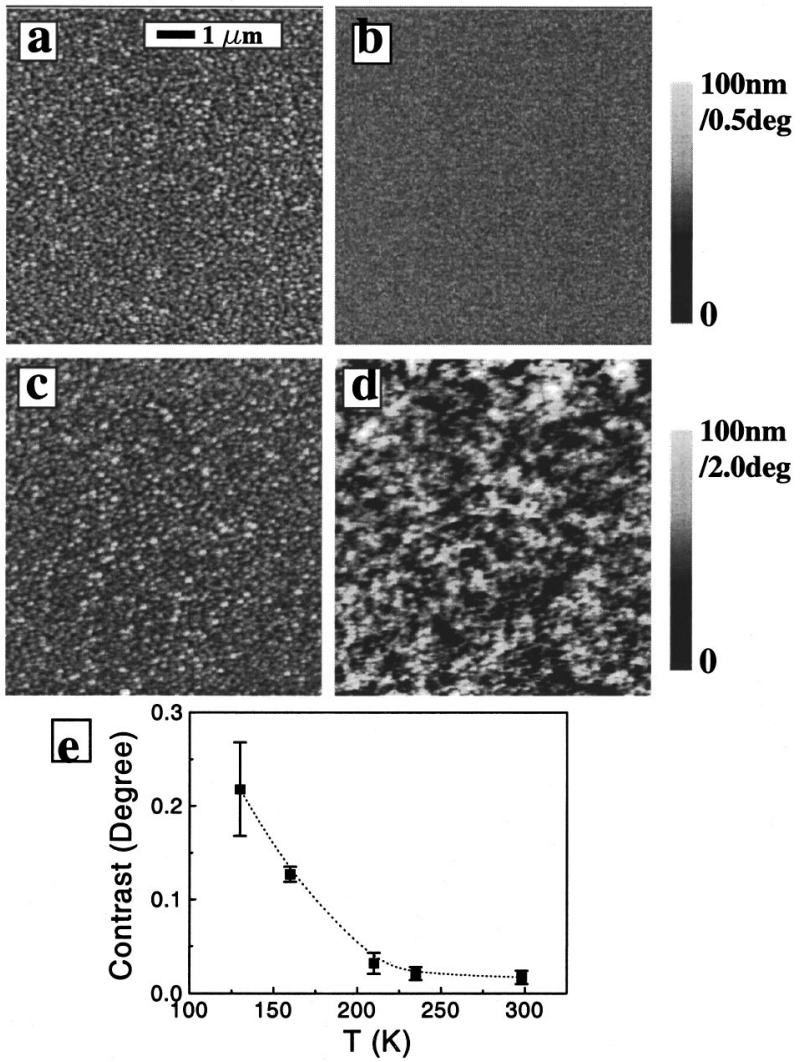

FIG. 3. Low temperature MFM images of the LCMO film on the LAO substrate. (a) and (c) show the topography of the sample surface, (b) and (d) are the corresponding magnetic structures. (a) and (b) are $8 \times 8 \mu \mathrm{m}^{2}$ images at room temperature and (c) and (d) are at $150 \mathrm{~K}$. The grayscales show $z$ ranges of height/phase for topographic/magnetic images. (e) is the relation between the average contrast in magnetic images and temperature. 

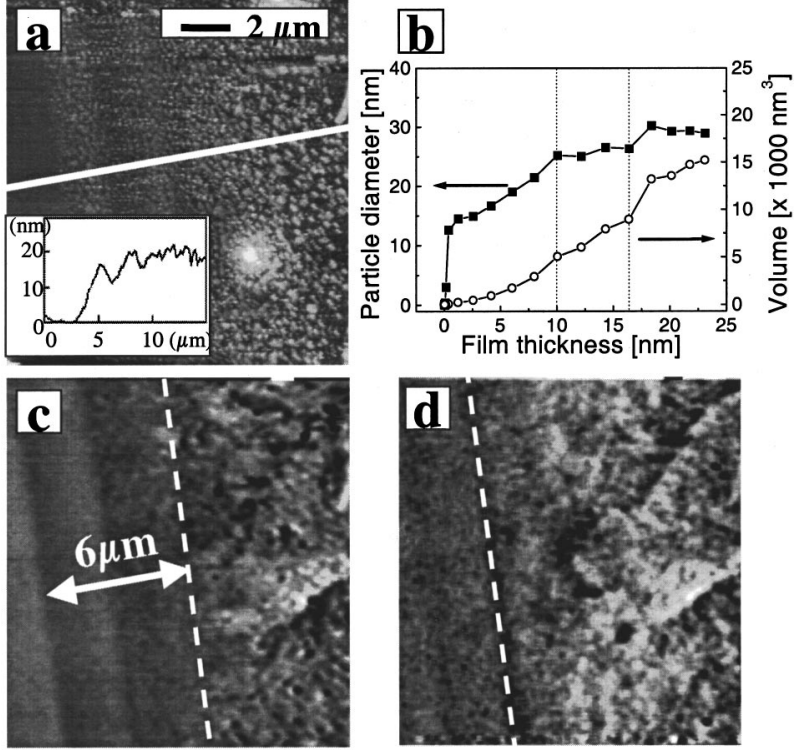

FIG. 4. Low temperature MFM images of a Co film $(20 \mathrm{~nm})$ on a $\mathrm{Si}$ substrate $\left(14 \times 14 \mu \mathrm{m}^{2}\right)$. (a) is the surface topography at the edge of the Co film. The Si substrate starts at the dark region. The inset is the crosssectional view along the white line. (b) shows the relation between the Co grain size and corresponding Co film thickness. (c) and (d) are the magnetic images corresponding to (a), at room temperature and $170 \mathrm{~K}$, respectively. Dashed lines indicate the expansion of the magnetic features with decreasing temperature. The $z$ range for these images is $100 \mathrm{~nm} / 2.5^{\circ}$

temperature. The images were obtained without an external magnetic field. Note the absence of any magnetic feature at room temperature as shown in Fig. 3(b), even as the sensitivity was increased to $0.5^{\circ}$. At $150 \mathrm{~K}$, however, strong magnetic contrast has emerged in Fig. 3(d), even as the sensitivity was reduced by a factor of 4 . The low temperature image was obtained far below the Curie temperature of $235 \mathrm{~K}$, which implies that the sample is near its maximum ferromagnetic behavior. The dark and bright areas can be interpreted as the magnetic poles for the in-plane magnetization. They are composed of several grains and roughly $500 \mathrm{~nm}$ to $1 \mu \mathrm{m}$. The contrast variation as a function of temperature is shown in Fig. 3(e). Very small contrast from measurement noise is observed at temperatures above $T_{c}$, but contrast intensifies as the temperature is lowered below $T_{c}$. The plot appears to vary more slowly than that suggested by the magnetization plot of Fig. 2. We suspect that this discrepancy between the two techniques may be due to the different measurement environments with regards to applied magnetic field. A more detailed result of successive imaging of the CMR material versus temperature and field will be published elsewhere.

We now apply our technique to study ferromagnetism in $\mathrm{Co} / \mathrm{Si}$ as a function of film thickness. Figure 4 shows images of an edge of the Co thin film deposited on $\mathrm{Si}(100)$. Figure 4(a) is the topography and the inset is the topographic profile from bare $\mathrm{Si}$ on the left to the $20 \mathrm{~nm}$ thick Co layer on the right. Figure 4(b) shows the film thickness dependence of Co grain sizes. Figures 4(c) and 4(d) show the corresponding magnetic structure of the same region at different temperatures.
The sample was made by molecular beam epitaxy growth using a shadow mask. The grains were measured with an in situ UHV scanning tunneling microscope. The edge of the Co film consists of a $6 \mu \mathrm{m}$ wide transition region where the thickness varied from the bare Si to the maximum Co film coverage of $20 \mathrm{~nm}$. In direct correspondence with the thickness, the grain size increases from less than a nanometer to about $30 \mathrm{~nm}$ at the thickest regions. The two dark stripes at the edge originate from an electrostatic effect or possibly arise from cobalt silicide formation. ${ }^{9}$ We excluded this additional electrostatic contribution by contact potential microscopy using the in situ UHV AFM when we measured the film thickness.

Figures 4(c) and 4(d) show the evolution with temperature of magnetic domain structures near the edge of the Co film. At room temperature, magnetic domain walls are seen at the lower right corner of the image, along with magnetic ripples. When the temperature is reduced to $170 \mathrm{~K}$, the domain wall contrast becomes more intense and a second domain wall forms in the upper right corner. More importantly, we find that the ripples have progressed to the left and approached the temperature invariant electrostatic lines. From Fig. 4(b), the diameter of Co grains in this region is around $25 \mathrm{~nm}$, which suggests that Co grains of about $25 \mathrm{~nm}$ are weakly ferromagnetic (or superparamagnetic) at room temperature but become ferromagnetic at $170 \mathrm{~K}$. We also observe some weak streak lines that occur when the tip changes magnetic features of fine particles with a low coercivity. Thus we can infer that the expansion of the magnetic ripple near the edge and the change in the magnetic contrast to the change of coercivity with temperature ${ }^{10}$ as well as the possible effects of superparamagnetism for fine grains.

These results demonstrate a conversion of a commercial MFM for operation at low temperatures. The operation is routine with no additional sample preparation. We demonstrated the efficacy of the technique to study paramagnetic to ferromagnetic transition in CMR materials, and superparamagnetic-ferromagnetic onset in Co grains. We believe that this technique opens new avenues for the investigations of low temperature micromagnetism.

\section{ACKNOWLEDGMENTS}

The authors thank J. B. Dottellis and R. Frizzell for useful discussions and assistance. This research was supported by UMCP NSF-MRSEC and NSF Career Award ECS9984797.

${ }^{1}$ Q. Lu, C.-C. Chen, and A. de Lozanne, Science 276, 2006 (1997)

${ }^{2}$ A. P. Volodin and M. V. Marchevsky, Ultramicroscopy 42, 757 (1992).

${ }^{3}$ C. W. Yuan et al., J. Vac. Sci. Technol. B 14, 1210 (1996).

${ }^{4}$ H. J. Hug et al., Rev. Sci. Instrum. 70, 3625 (1999).

${ }^{5}$ MultiMode ${ }^{\mathrm{TM}}$, Digital Instruments, Inc., Santa Barbara, CA.

${ }^{6}$ M. Dreyer, R. D. Gomez, and I. D. Mayergoyz, IEEE Trans. Magn. (to be published).

${ }^{7}$ C. J. Lu et al., J. Appl. Phys. 88, 4032 (2000).

${ }^{8}$ P. Schiffer et al., Phys. Rev. Lett. 75, 3336 (1995).

${ }^{9}$ M. Dreyer and R. D. Gomez, J. Appl. Phys. (to be published).

${ }^{10}$ B. D. Cullity, Introduction to Magnetic Materials (Addison-Wesley, Reading, MA, 1972), pp. 410-418. 